\title{
GENERATING MUSIC THERAPY USING DEEP LEARNING AND REINFORCEMENT LEARNING
}

\author{
Sarthak Sengupta \\ Department of Computer Science and Engineering \\ IILM College of Engineering and Technology, \\ Greater Noida, Uttar Pradesh, India
}

\begin{abstract}
This paper describes a software system with an objective to develop a system to generate personalized and effective sound/music therapy mechanism using Deep Learning and Reinforcement Learning to help the users:

- Cure mental health problems

- Improve mental and physical health

The project also aims to provide a seamless feedback mechanism which would introduce a sense of participation and control of the music therapy which leads to the formation of a better understanding between the system and the users.
\end{abstract}

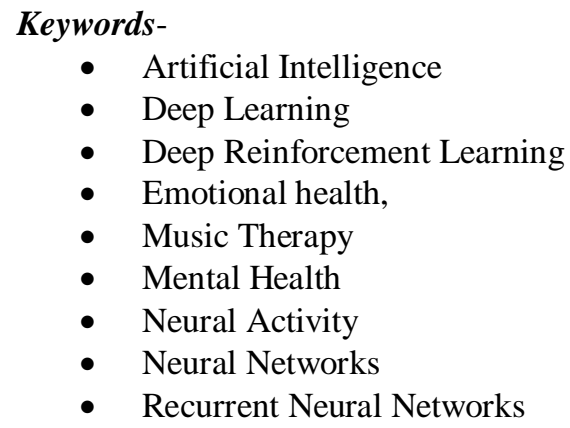

\section{INTRODUCTION}

Sound / Music therapy uses aspects of music to improve your physical, mental and emotional health and well-being. The person being treated partakes in the experience with a trained music therapy practitioner. Music therapy may involve:

- listening to music

- $\quad$ singing along to the music

- moving to the beat of the music

- meditating

- playing an instrument ${ }^{[10]}$

The therapeutic sense of music can be in part explained by its cultural role in facilitating social learning and mental and emotional well-being. However, several studies have indicated that rhythmic entertainment of motor function can actively facilitate the recovery of movement in patients with stroke, Parkinson's disease, cerebral palsy and traumatic brain injury [2]

Studies of people with memory disorders, such as Alzheimer's disease, indicate that neural memory traces built through music are deeply and strongly ingrained and more resilient to neurodegenerative influences. Findings from individually

\author{
Dr. Anuradha Konidena \\ Department of Computer Science and Engineering \\ IILM College of Engineering and Technology, \\ Greater Noida, Uttar Pradesh, India
}

randomized trials suggest that music therapy is accepted by people with depression and is associated with improvements in mood disorders. ${ }^{[2]}$

\section{BACKGROUND}

Music generation was earlier using deep learning has involved training a Recurrent Neural Networks (RNN) to learn to predict the next note in the melody. Often, this RNN is implemented using a Long Short Term Memory (LSTM) network.

Basic RNNs are neural networks in which nodes are organized into successive layers. Each node in a given layer is connected to another node in the next successive layer with a directed connection. Each node has a time independent real activation value. Each connection (synapse) has a modifiable weight. Nodes are either input nodes (receiving data from outside the network), output nodes (yielding results to the outer system), or hidden nodes (that modify the data en route from input to output). ${ }^{[3]}$

LSTMs are neural networks in which each of the recurrent cells learns to control the storage of the information through the use of multiple gates namely input gate, output gate, and forget gate. The input gate and output gate gates control whether the information is able to flow into and out of the cell, and the forget gate controls whether or not the contents of the cell should be reset. These characteristics make LSTMs better at learning long-term dependencies in the data and makes it capable of adapting more rapidly and dynamically to new data.

Reinforcement learning (RL) is a sub-division of machine learning which involves software agents to make decisions and take actions in the given environment to maximize the accumulative reward which includes not only the immediate reward but also the rewards to be gained in the future.

In RL, an agent interacts with an environment. Given the state of the environment at time $\mathrm{t}, \mathrm{s}_{\mathrm{t}}$, the RL agent takes an action at according to its policy $\pi\left(\mathrm{a}_{\mathrm{t}} \mid \mathrm{s}_{\mathrm{t}}\right)$, receives a reward $\mathrm{r}\left(\mathrm{s}_{\mathrm{t}}, \mathrm{a}_{\mathrm{t}}\right)$, and the environment transitions to a new state, $\mathrm{s}_{\mathrm{t}}+1$. The agent's goal is to maximize reward over a sequence of actions, with a discount factor of $\gamma$ applied to future rewards. ${ }^{[3]}$

Music Therapy is an established health profession in which music is used within a therapeutic relationship to address physical, emotional, cognitive, and social needs of individuals. ${ }^{[6]}$ 


\section{International Journal of Engineering Applied Sciences and Technology, 2020 Vol. 4, Issue 12, ISSN No. 2455-2143, Pages 502-505 \\ Published Online April 2020 in IJEAST (http://www.ijeast.com)}

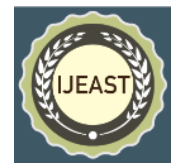

Music therapy is a burgeoning field. Certified music therapists are usually accomplished musicians who have attained deep knowledge of how music can evoke emotional responses to relax or stimulate people or help them heal their health issues. This knowledge is generally combined with their familiarity with a wide variety of musical styles to find the specific kind or genre of the music that can get you into a desirable state such as in meditation. ${ }^{[9]}$

\section{RELATED WORKS}

Jaques et al.(2016) in their paper Tuning Recurrent Neural Networks with Reinforcement Learning ${ }^{[3]}$ propose and explains Recurrent Neural network (RNN) can be tuned or improved using reinforcement learning in a way such that it can learn new musical concept without overlooking the achieved information from the initial training over the music compositions. Long Short Term Memory (LSTM) trained on data (RNN) is used to supply the initial weights for three networks in RL Tuner: the Q-network and Target Q-network in the DQN algorithm as described in Section 2.1, and a Reward RNN. The Reward RNN is held fixed and used to supply part of the reward value used in training.

To express musical composition as an RL problem, generating the next note in the composition is considered as taking an action. The state of the environment $\mathrm{s}$ consists of both the notes placed in the composition so far and the internal state of the LSTM cells of both the Q-network and the Reward RNN. To calculate the reward, probabilities learned from the training data are combined with knowledge of music theory. A set of music theory based rules are defined to apply constraints on the melody that the model is composing through a reward signal in the model. A constant is used to control the emphasis placed on the music theory reward.

Leubner et al.(2017) in their paper Reviewing the Effectiveness of Music Interventions in Treating Depression $^{[5]}$ discuss how the Music and Music therapy can cater to helping the people cure depression. Depression is one of the most common mood disorders, resulting in a hindered social function, reduced quality of life and increased mortality. Music interventions have a potential to become an alternative for depression therapy but the number of up-to-date research literature is quite limited. The aim of this research was to differentiate the impact of the therapeutic applications of music used in various experiments. The factors such as length of the trial, number of sessions, participants' age, kind of music, active or passive participation and single- or group setting were used as critical parameters for the experiments. Activities like questionnaires about participants' confidence, self-esteem or motivation were used to confirm further improvements after music treatment.

Hohmann et al.(2017) in their paper Effects of music therapy and music-based interventions in the treatment of substance use disorder ${ }^{[7]}$ explored how Music therapy (MT) and music-based interventions (MBIs) are increasingly used for the treatment of substance use disorders (SUD).

meta-analysis was planned, but due to the diversity of the quantitative studies, effect sizes were not computed. The beneficial effects of Music Therapy on motivational and emotional outcomes, participation, locus of control, and perceived helpfulness were discovered, but results were found to be inconsistent across studies.

Trimble et al.(2017) in their work Music and the brain: the neuroscience of music and musical appreciation ${ }^{[2]}$ discusses the music being a potential method of therapy and considered to be the means of accessing and stimulating or impacting the specific cerebral circuits. Association between musical creativity and psychopathology is an interesting perspective.

\section{Methodology}

1) The initial step of the process is to train a Recurrent Neural Network (RNN) on a large variety of existing music therapy mechanisms or compositions to extract insight and learn how to produce effective original music therapy.

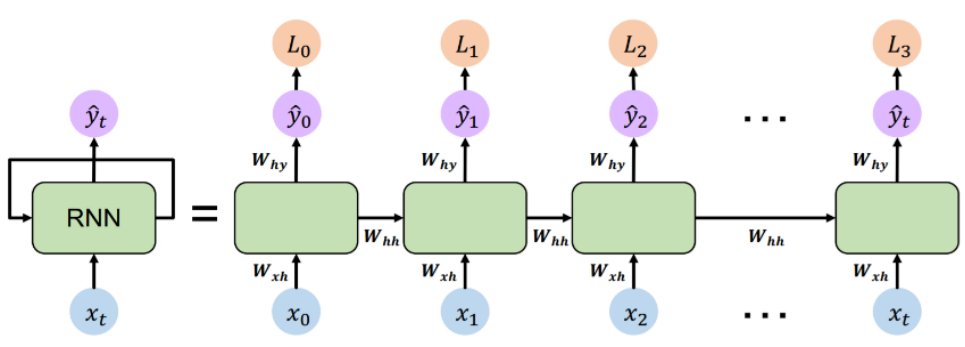

Figure 4.1 source: http://introtodeeplearning.com/

2) The RNN units during training would receive music notes/audio samples $\left(\mathrm{x}_{\mathrm{t}}\right)$ in the form of midi and used to produce the next note in the sequence $\left(\mathrm{y}_{\mathrm{t}}\right)$.

3) The trained the RNN model would be used to produce music therapy based on the preferences and mental state of the user.

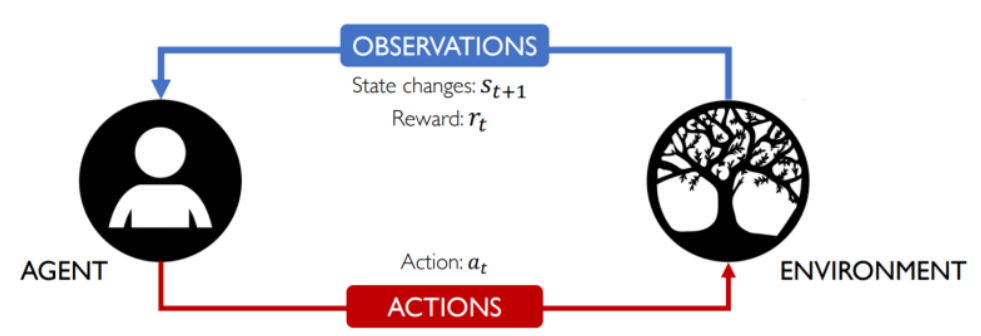

Figure 4.2 source: http://introtodeeplearning.com/

4) The system accepts real-time feedback from the users about its effectiveness and impact through simple 


\section{International Journal of Engineering Applied Sciences and Technology, 2020 \\ Vol. 4, Issue 12, ISSN No. 2455-2143, Pages 502-505 \\ Published Online April 2020 in IJEAST (http://www.ijeast.com)}

actions like scrolling up and down without causing any disturbance through interaction.

5) The received feedback is used in reinforcement learning to understand the preferences, improve the model and personalize it for the users

6) Here the Agent would receive the feedback from the user and takes action in order to improve the model.

7) The model uses the feedback received from the user and derives reward for deep reinforcement learning to perform further actions accordingly.

8) This improved model is used to further generate further notes in the composition of music therapy.

9) The system can run this process indefinitely and improves substantially with time.

\section{CONTROL FLOW OF THE SYSTEM}

1) System Authentication Flow.

i. Users would be able to sign up to the system to save their minimal profile information and preferences.

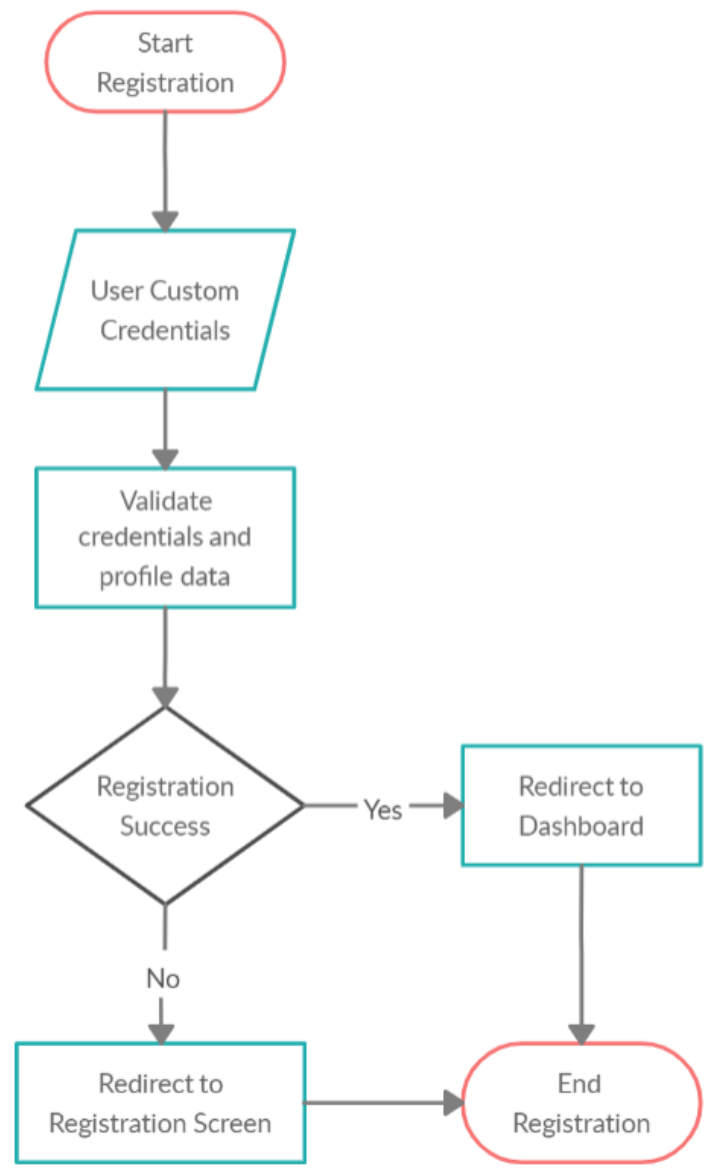

Figure 5.1

ii. Users will be able to sign in to the system securely with their custom authentication credentials.

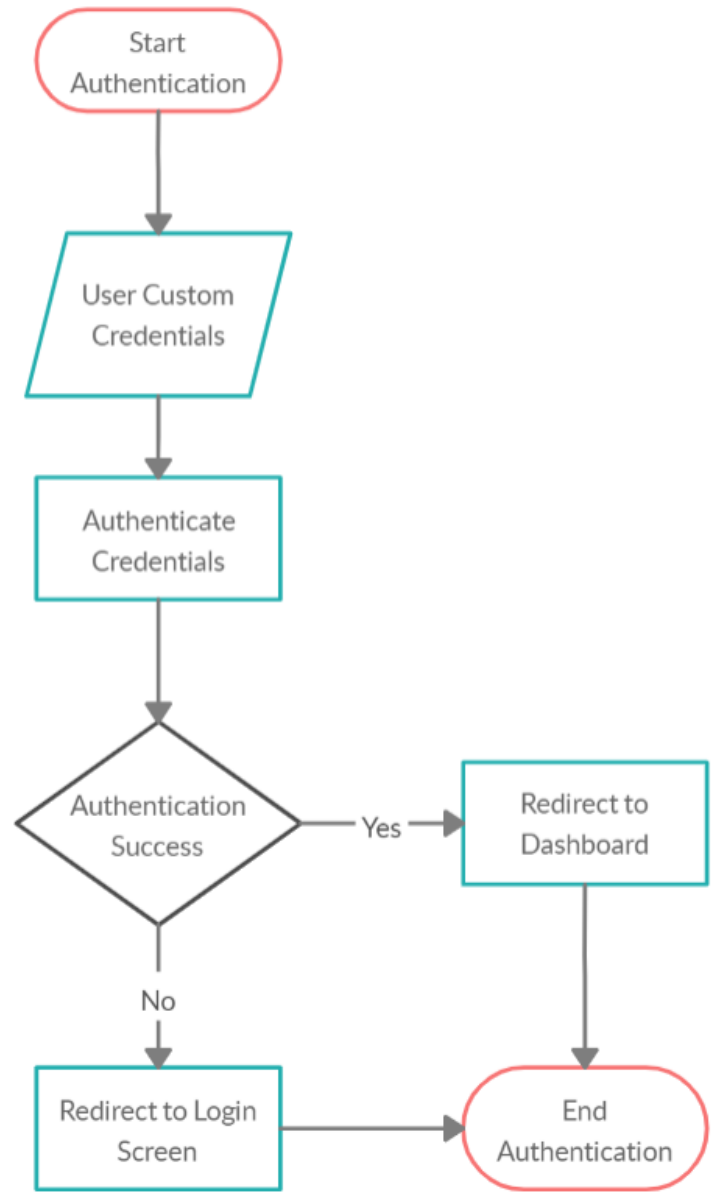

Figure 5.2

2) Main System Flow

i. The user receives Music Therapy audio samples produced from the machine learning model which was initially trained on the large data set of a variety of music therapy and then continue to improve with reinforcement learning.

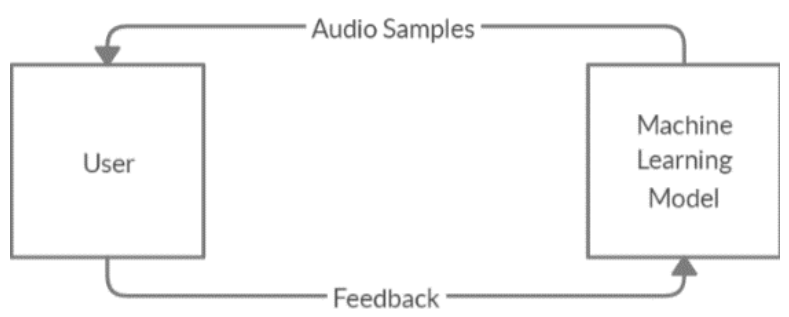

Figure 5.3

ii. The user is provided with an interface accepting feedback in real-time and sending feedback to the machine learning model. 


\section{International Journal of Engineering Applied Sciences and Technology, 2020 Vol. 4, Issue 12, ISSN No. 2455-2143, Pages 502-505 \\ Published Online April 2020 in IJEAST (http://www.ijeast.com)}

iii. The model uses the feedback received from the user and derives reward/punishment for deep reinforcement learning and performs further actions accordingly.

\section{STUdies ANd FInDINGS}

The RNN is tested on a variety of hyperparameters chosen randomly and found to be successful with the following values

1) System Parameters

i. Algorithm: Deep Q Network

ii. Scalar Value measuring the importance of music theory in the compositions: 0.7

2) RNN Hyperparameters

i. Initial Learning Rate $=0.5$,

ii. Learning Rate Decay Steps $=1000$,

iii. Learning Rate Decay Rate $=0.85$,

iv. Number of Layers in $\mathrm{RNN}=2$

v. RNN Layer Sizes $=512,512$

vi. Skip First N Losses $=32$,

vii. Exponentially Decay Learning Rate $=$ True

3) Deep Q Network Hyperparameters

i. Random Action Probability $=0.3$

ii. Training Every nth $=5$

iii. Minibatch size $=32$

iv. Discount rate $=0.5$

v. Max Experience $=100000$

vi. Target Network Update Rate $=0.01$

VII. RESULTS

1) The resulted system is able to generate personalized music therapy by adapting

2) The functioning demo of the system and detailed documents can be viewed at the following link: https://drive.google.com/drive/folders/1yYe4-ywXNVdADDi74IzuCBG0jmPSLhm?usp=sharing

\section{LIMITATIONS}

1) The system would not be able to help users who are hearing-impaired.

2) The system would not help users in noisy environments.

3) The system in some cases needs to be accompanied by other kinds of treatments

\section{CONCLusions}

1) The proposed model can be used by medical practitioners to keep their patients calm and reduce their sense of pain.

2) The system can be used in academics to improve concentration and memory of the users which would also lead to better performance of students and employees
3) This Innovative health practice not only ensures Good Health and Well-being of a human being but also improves cognitive functioning which leads to Economic growth of society.

4) The system can also be monetized for usage above a certain limit and with the inclusion of some exclusive features specifically for certain professions and industries.

\section{REFERENCES}

[1] Adam Roberts and Anna Huang and Curtis Hawthorne and Jacob Howcroft and James Wexler and Leon Hong and Monica Dinculescu, 2019, Approachable music composition with machine learning at scale, arXiv:1907.06637

[2] Michael Trimble and Dale Hesdorffer, 2017, Music and the brain: the neuroscience of and musical appreciation, PMCID: PMC5618809

[3] Natasha Jaques and Shixiang Gu and Dzmitry Bahdanau and Jose Miguel Hernandez Lobato and Richard E. Turner and Doug Eck, 2016, Tuning Recurrent Neural Networks With Reinforcement Learning, arXiv:1611.02796v2

[4] Sutton et al., 1999, Policy gradient methods for reinforcement learning with function approximation. In NIPS, volume 99, pp. 1057-1063

[5] Daniel Leubner and Thilo Hinterberger, 2017, Reviewing the Effectiveness of Music Interventions in Treating Depression

[6] Music Therapy Organization Quotes, https://www.musictherapy.org/about/quotes/

[7] Louisa Hohmann and Joke Bradt and Thomas Stegemann and Stefan Koelsch, 2017, Effects of music therapy and music-based interventions in the treatment of substance use disorders

[8] The British Academy of Sound Therapy, https://www.britishacademyofsoundtherapy.com

[9] How music can help you heal, Harvard Health Publishing, 2016

[10] The Uses and Benefits of Music Therapy, www.healthline.com/health/sound-healing

[11] Magenta (Tensorflow), https://magenta.tensorflow.org/

[12] Van Hasselt, Guez, and Silver, 2015, Deep reinforcement learning with double Q-learning. CoRR, abs/1509.06461. 